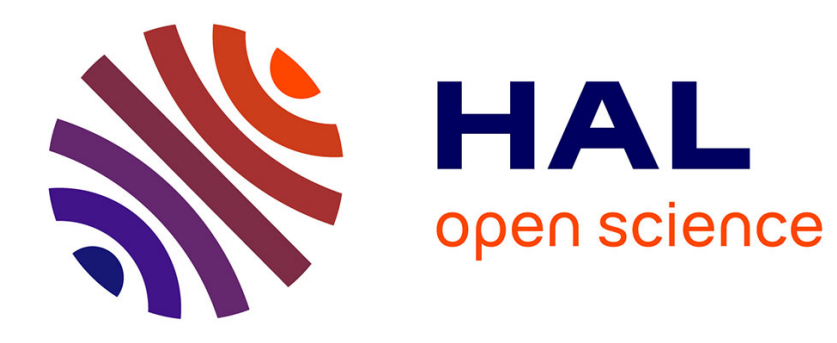

\title{
Properties of Tiny Braids and the Associated Commuting Graph
}

\author{
Azeem Haider, Usman Ali, Moin A Ansari
}

\section{To cite this version:}

Azeem Haider, Usman Ali, Moin A Ansari. Properties of Tiny Braids and the Associated Commuting Graph. Journal of Algebraic Combinatorics, 2019, 10.1007/s10801-019-00923-5 . hal-02408846

\section{HAL Id: hal-02408846 https://hal.sorbonne-universite.fr/hal-02408846}

Submitted on 13 Dec 2019

HAL is a multi-disciplinary open access archive for the deposit and dissemination of scientific research documents, whether they are published or not. The documents may come from teaching and research institutions in France or abroad, or from public or private research centers.
L'archive ouverte pluridisciplinaire HAL, est destinée au dépôt et à la diffusion de documents scientifiques de niveau recherche, publiés ou non, émanant des établissements d'enseignement et de recherche français ou étrangers, des laboratoires publics ou privés. 


\title{
Properties of Tiny Braids and the Associated Commuting Graph
}

\author{
Azeem Haider ${ }^{1}$, Usman Ali $^{2}$, Moin A. Ansari ${ }^{3}$ \\ ${ }^{1,3}$ Department of Mathematics, Jazan University, Jazan, Saudi Arabia \\ E-mail: aahaider@jazanu.edu.sa,maansari@jazanu.edu.sa \\ ${ }^{2}$ Centre for Advanced Studies in Pure and Applied Mathematics, Bahauddin \\ Zakaria University, Multan, Pakistan \\ ${ }^{2}$ Institut de Mathématiques de Jussieu-Paris Rive Gauche, Paris, France \\ E-mail: uali@bzu.edu.pk
}

\begin{abstract}
In this text, we focus on a subset (called the set of tiny braids) of factors of the Garside braid and generalize some known results related to tiny braids. These generalized results, along with some combinatorics, strengthen the existing relationship between this subset and Fibonacci numbers. We also associate a commuting graph with the subset and explore its fundamental identities, including its order, diameter, girth and degree-related properties.
\end{abstract}

Keywords: Fibonacci numbers; centralizer; commuting graph; positive braid; tiny braid.

2000 Mathematics Subject Classification: 11B39, 05A15, 05 A05.

\section{Introduction}

The monoid of positive $n$-braids $\mathcal{B}_{n}^{+}$is defined in [3] as

$$
\mathcal{B}_{n}^{+}=\left\langle\sigma_{1}, \sigma_{2}, \cdots, \sigma_{n-1}: \begin{array}{l}
\sigma_{i+1} \sigma_{i} \sigma_{i+1}=\sigma_{i} \sigma_{i+1} \sigma_{i} \\
\sigma_{i} \sigma_{j}=\sigma_{j} \sigma_{i} \text { for }|i-j| \geq 2
\end{array}\right\rangle .
$$

In fact, a positive braid $\alpha$ is a class of words in the set of generators $\left\{\sigma_{1}, \sigma_{2}, \ldots, \sigma_{n-1}\right\}$ :

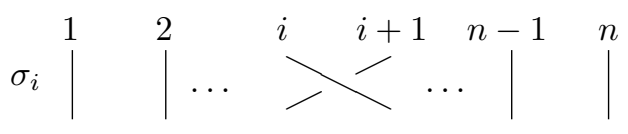


The monoid $\mathcal{B}_{n}^{+}$canonically embedds in the braid group $\mathcal{B}_{n}$, which is defined by the same presentation as $\mathcal{B}_{n}^{+}$, but considered as a presentation of a group. The monoid $\mathcal{B}_{n}^{+}$is embedded in $\mathcal{B}_{n+1}^{+}$, and two braids $\alpha, \beta \in \mathcal{B}_{n}^{+}$commute in $\mathcal{B}_{n+1}^{+}$if and only if $\alpha$ and $\beta$ commute in $\mathcal{B}_{n}^{+}$.

Three kinds of divisors of $\alpha \in \mathcal{B}_{n}^{+}$are defined as factors of $\alpha:(\gamma \mid \alpha)$, $\operatorname{Div}(\alpha)=\left\{\gamma \in \mathcal{B}_{n}^{+}\right.$: there exist $\left.\delta, \varepsilon \in \mathcal{B}_{n}^{+}, \alpha=\delta \gamma \varepsilon\right\}$; left divisors of $\alpha$ $\left(\left.\gamma\right|_{L} \alpha\right), \operatorname{Div}_{L}(\alpha)=\left\{\gamma \in \mathcal{B}_{n}^{+}\right.$: there exists $\left.\varepsilon \in \mathcal{B}_{n}^{+}, \alpha=\gamma \varepsilon\right\}$; and right divisors of $\alpha\left(\left.\gamma\right|_{R} \alpha\right), \operatorname{Div}_{R}(\alpha)=\left\{\gamma \in \mathcal{B}_{n}^{+}\right.$: there exists $\left.\delta \in \mathcal{B}_{n}^{+}, \alpha=\delta \gamma\right\}$. Clearly, $\operatorname{Div}_{L}(\alpha) \cup \operatorname{Div}_{R}(\alpha) \subseteq \operatorname{Div}(\alpha)$.

Let $\mathcal{T} \mathcal{B}_{n}$ be the set of all positive braids in which a letter $\sigma_{i}$ occurs at most once (see [4]). Clearly, any word representing a braid in the set $\mathcal{T} \mathcal{B}_{n}$ must contain $\sigma_{i}$ at most once. The set $\mathcal{T} \mathcal{B}_{n}$ is a proper subset of $\operatorname{Div}\left(\Delta_{n}\right)$, where

$$
\Delta_{n}=\sigma_{1}\left(\sigma_{2} \sigma_{1}\right) \cdots\left(\sigma_{n-1} \sigma_{n-2} \cdots \sigma_{2} \sigma_{1}\right)
$$

is the Garside braid (see [8]). The set $\operatorname{Div}\left(\Delta_{n}\right)$ plays a vital role in the solutions to the word and conjugacy problems given in [8]. In the solution to the latter problem, the factors of the braid $\Delta_{n}$ are used to generate an invariant (called the summit set) of a conjugacy class. Note that in the classical literature on braid theory, every factor of the Garside braid is known as a simple braid, but in $[4,5]$, the elements from the set $\mathcal{T} \mathcal{B}_{n}$ are called simple braids. Previous articles $[1,2]$ follow the same naming convention for the braids in $\mathcal{T} \mathcal{B}_{n}$. To avoid confusion, we call an element from the set $\mathcal{T} \mathcal{B}_{n}$ a tiny braid instead of a simple braid.

[5] shows that the number of elements in the set $\mathcal{T} \mathcal{B}_{n}$ is a Fibonacci number $F_{2 n-1}$, where $\left(F_{0}, F_{1}, F_{2}, F_{3}, F_{4}, F_{5} \ldots\right)=(0,1,1,2,3,5, \ldots)$. For further details regarding and the motivation behind defining the set $\mathcal{T} \mathcal{B}_{n}$, see [4] or [5]. A subset of the centralizer of an element $\alpha \in \mathcal{T} \mathcal{B}_{n}$ is defined in [2] as follows:

$$
C_{n}(\alpha)=\left\{\gamma \in \mathcal{T B}_{n}: \alpha \gamma=\gamma \alpha\right\}
$$

An element $\alpha \in \mathcal{T B}_{n}$ is said to have a trivial tiny centralizer (in [2], the set $C_{n}(\alpha)$ is called a simple centralizer) if

$$
C_{n}(\alpha)=\{e, \alpha\}
$$

Let $I_{n}$ be the set of all $\alpha \in \mathcal{T} \mathcal{B}_{n}$ that have a trivial tiny centralizer. In the first part of this article, we discuss the different properties of tiny centralizers and generalize a few of the results. Note that throughout this article, we use letters $h, i, j, k, l, m$ to denote positive integers (for further details on braids, see [10]).

For graph $G$, we denote the set of vertices by $V(G)$, the set of edges by $E(G)$, the degree of a vertex $v \in V(G)$ by $\operatorname{deg}(v)$ and the number of vertices (order) of a graph $G$ by $|V(G)|$. Graph $K_{n}$ denotes the complete 
graph on $n$ vertices. The maximum value of the shortest distance between any two vertices of $G$ is called its diameter. Graph $G$ is said to be connected if a path exists between any two vertices of $G$. The girth of $\mathrm{G}$, denoted by $\operatorname{girth}(G)$, is the length of its shortest nontrivial cycle, if it exists. The minimum and maximum degrees of a vertex in $G$ are denoted by $\delta(G)$ and $\Delta(G)$, respectively. For further details on graph theory, see, e.g., [11].

The commuting graph $\Gamma(H)$ associated with a finite subset $H$ of group $G$ is a simple graph whose vertices are the elements of $H \backslash\{e\}$. An edge exists between $g$ and $h$ if and only if $g h=h g$ in $G$ (see $[6,7,9]$ ).

For $n \geq 4$, we define a commuting graph $\Gamma\left(\mathcal{T} \mathcal{B}_{n}\right)$ associated with the set $\mathcal{T B}_{n}$, the vertex set of which is the set $\mathcal{T} \mathcal{B}_{n} \backslash\left(I_{n} \cup\{e\}\right)$; that is, $V\left(\Gamma\left(\mathcal{T} \mathcal{B}_{n}\right)\right)=\mathcal{T} \mathcal{B}_{n} \backslash\left(I_{n} \cup\{e\}\right)$. An edge exists between two distinct vertices if and only if they commute in $\mathcal{B}_{n}^{+}$. In fact, the graph $\Gamma\left(\mathcal{T} \mathcal{B}_{n}\right)$ defined here is the major component of the commuting graph defined in [2], where the vertex set is the entire set $\mathcal{T} \mathcal{B}_{n}$. Graphs $\Gamma\left(\mathcal{T} \mathcal{B}_{4}\right)=K_{3}$ and $\Gamma\left(\mathcal{T} \mathcal{B}_{5}\right)$ are shown in Figure 1. Fifty-two vertices exist in $\Gamma\left(\mathcal{T} \mathcal{B}_{6}\right)$, and the graph

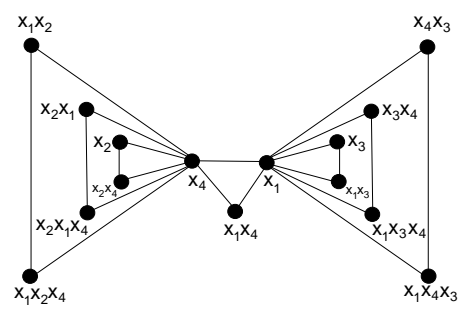

Figure 1: $\Gamma\left(\mathcal{T} \mathcal{B}_{5}\right)$

structure becomes more complex for $n \geq 7$.

In the second section of this article, we focus on the graphical aspect of this association and investigate some properties of the commuting graph $\Gamma\left(\mathcal{T} \mathcal{B}_{n}\right)$, such as its order, connectedness, diameter, girth and degree-related results.

To develop our text, we need the following known results:

Theorem 1.1. [1] If $\alpha \in \mathcal{T B}_{n}$ with $\sigma_{i} \mid \alpha$ for all $1<i<n-1$, then the tiny centralizer $C_{n}(\alpha)=\{e, \alpha\}$.

Lemma 1.2. [2] If $\alpha \in \mathcal{T} \mathcal{B}_{n}$ and $\sigma_{n-1} \mid \alpha$, then either $\left.\sigma_{n-1}\right|_{R} \alpha$ or $\left.\sigma_{n-1}\right|_{L} \alpha$.

Lemma 1.3. [2] If $\beta\left(\gamma_{1} \gamma_{2}\right)=\left(\gamma_{1} \gamma_{2}\right) \beta$ and $\beta \gamma_{1}=\gamma_{1} \beta$ or $\beta \gamma_{2}=\gamma_{2} \beta$, then $\beta \gamma_{2}=\gamma_{2} \beta$ or $\beta \gamma_{1}=\gamma_{1} \beta$, respectively.

Lemma 1.4. [2] If $\beta \in \mathcal{T B}_{n-1}$ and $\alpha \in \mathcal{T H}_{n}$ such that $\sigma_{n-2} \mid \beta$ and $\sigma_{n-1} \mid \alpha$, then $\beta \alpha \neq \alpha \beta$. 


\section{Centralizer of Tiny Braids}

We define a set $\mathcal{T} \mathcal{B}_{(0, n)}=\mathcal{T} \mathcal{B}_{n}$. Generally,

$$
\mathcal{T B}_{(i, j)}=\left\{\alpha \in \mathcal{T B}_{n}: \sigma_{k} \nmid \alpha \text { for all } k \leq i \text { and } k \geq j\right\}, i \leq j<n .
$$

Correspondingly, we define, for any $\alpha \in \mathcal{T} \mathcal{B}_{n}$, the set $C_{(i, j)}(\alpha)=\{\gamma \in$ $\left.\mathcal{T B}_{(i, j)}: \alpha \gamma=\gamma \alpha\right\}$.

We rewrite the following lemma from [2] using the above construction.

Lemma 2.1. [2] If $\beta \in \mathcal{T B}_{i}, i>1$ and $\sigma_{i-1} \mid \beta$, then $\gamma \in C_{n}(\beta)$ if and only if $\gamma=\gamma_{1} \gamma_{2}$, where $\gamma_{1} \in C_{i}(\beta)$ and $\gamma_{2} \in C_{(i, n)}(\beta)=\mathcal{T} \mathcal{B}_{(i, n)}$.

Now, consider the set $C_{0}(\alpha)=\{e\}$ for any $\alpha \in \mathcal{T B}_{n}, \mathcal{T} \mathcal{B}_{0}=\{e\}$ and $\mathcal{T B}_{(n, n)}=\{e\}$. Due to the symmetric nature of $\sigma_{i}$ and $\sigma_{n-i}$ in set $\mathcal{T} \mathcal{B}_{n}$, and using Lemma 2.1, we obtain the following result.

Lemma 2.2. If $\beta \in \mathcal{T B}_{(i, n)}$ and $\sigma_{i+1} \mid \beta$, then $\gamma \in C_{n}(\beta)$ if and only if $\gamma=\gamma_{1} \gamma_{2}$, where $\gamma_{1} \in C_{i}(\beta)=\mathcal{T} \mathcal{B}_{i}$ and $\gamma_{2} \in C_{(i, n)}(\beta)$.

Lemma 2.1 and Lemma 2.2 lead to the following result.

Theorem 2.3. For any $\beta \in \mathcal{T B}_{n}$, if $i+1=\min \left\{k: \sigma_{k} \mid \beta\right\}$ and $j-1=$ $\max \left\{k: \sigma_{k} \mid \beta\right\}$ with $i<j<n$, then $\gamma \in C_{n}(\beta)$ if and only if $\gamma=\gamma_{1} \gamma_{2} \gamma_{3}$, where $\gamma_{1} \in \mathcal{T}_{\mathcal{B}_{i}}, \gamma_{2} \in C_{(i, j)}(\beta)$ and $\gamma_{3} \in \mathcal{T H}_{(j, n)}$. This can be written symbolically as

$$
\begin{aligned}
C_{n}(\beta) & =\mathcal{T B}_{i} \times C_{(i, j)}(\beta) \times \mathcal{T B}_{(j, n)} \\
& =\left\{\gamma_{1} \gamma_{2} \gamma_{3}: \gamma_{1} \in \mathcal{T} \mathcal{B}_{i}, \gamma_{2} \in C_{(i, j)}(\beta), \gamma_{3} \in \mathcal{T B}_{(j, n)}\right\}
\end{aligned}
$$

Proof. Because $\beta \in \mathcal{T B}_{j}$ when $\sigma_{j-1} \mid \beta$, by Lemma 2.1, $\gamma \in C_{n}(\beta)$ if and only if $\gamma=\gamma^{\prime} \gamma_{3}$, where $\gamma^{\prime} \in C_{j}(\beta)$ and $\gamma_{3} \in C_{(j, n)}(\beta)=\mathcal{T B}_{(j, n)}$. Additionally, the given conditions indicate that $\beta \in \mathcal{T} \mathcal{B}_{(i, j)}$, with $\sigma_{i+1} \mid \beta$. Therefore, by Lemma $2.2, \gamma^{\prime} \in C_{j}(\beta)$ if and only if $\gamma^{\prime}=\gamma_{1} \gamma_{2}$, where $\gamma_{1} \in C_{i}(\beta)=\mathcal{T} \mathcal{B}_{i}$ and $\gamma_{2} \in C_{(i, j)}(\beta)$.

For any $\alpha \in \mathcal{T} \mathcal{B}_{n},\left|C_{n}(\alpha)\right|$ denotes the number of elements in the tiny centralizer $C_{n}(\alpha)$. [5] shows that $\left|\mathcal{T} \mathcal{B}_{n}\right|=F_{2 n-1}$. Furthermore, $\left|\mathcal{T} \mathcal{B}_{(j, n)}\right|=$ $\left|\mathcal{T} \mathcal{B}_{n-j}\right|=F_{2(n-j)-1}$, as per our consideration, we take $\left|\mathcal{T} \mathcal{B}_{0}\right|=|\{e\}|=$ $F_{-1}=1$. As a consequence, we have the following corollary:

Corollary 2.4. For any $\beta \in \mathcal{T} \mathcal{B}_{n}$, if $i+1=\min \left\{k: \sigma_{k} \mid \beta\right\}$ and $j-1=$ $\max \left\{k: \sigma_{k} \mid \beta\right\}$, with $i<j<n$, then

$$
\left|C_{n}(\beta)\right|=\left|C_{(i, j)}(\beta)\right| F_{2 i-1} F_{2(n-j)-1} .
$$


Theorem 2.3 and Corollary 2.4 are generalizations of the previous results found in [2], which were obtained only for the generators of $\mathcal{B}_{n}^{+}$.

We define a segment set in set $\mathcal{T} \mathcal{B}_{n}$ as

$$
\left[\sigma_{i}, \sigma_{i+j}\right]=\left\{\alpha \in \mathcal{T B}_{n}: \sigma_{k} \mid \alpha \text { if and only if } i \leq k \leq i+j\right\} .
$$

The construction of these segments is significant for set $\mathcal{T} \mathcal{B}_{n}$. A complete characterization of $C_{n}\left(\sigma_{i}\right)$ is given in [2], which shows that $\gamma \in C_{n}\left(\sigma_{i}\right)$ if and only if $\sigma_{k} \nmid \gamma$ for any $k$ satisfying $|k-i|=1$. As a generalization, we characterize the tiny centralizer $C_{n}(\alpha)$ for $\alpha \in\left[\sigma_{i}, \sigma_{i+j}\right]$.

Theorem 2.5. If $\alpha \in\left[\sigma_{l}, \sigma_{k}\right] \subset \mathcal{T} \mathcal{B}_{n}, l \leq k<n$, then

$$
C_{n}(\alpha)=\mathcal{T B}_{l-1} \times\{e, \alpha\} \times \mathcal{T B}_{(k+1, n)} .
$$

Moreover,

$$
\left|C_{n}(\alpha)\right|=2 F_{2 l-3} F_{2(n-k)-3} .
$$

Proof. Theorem 1.1 states that $C_{n}(\alpha)=\{e, \alpha\}$ for any $\alpha \in\left[\sigma_{1}, \sigma_{n-1}\right]$. Therefore, for any $\alpha \in\left[\sigma_{l}, \sigma_{k}\right]$, we have $C_{(l-1, k+1)}(\alpha)=\{e, \alpha\}$ in the set $\mathcal{T} \mathcal{B}_{(l-1, k+1)}$. By substituting $i-1=l$ and $j+1=k$ into Theorem 2.3, we obtain

$$
C_{n}(\alpha)=\mathcal{T B}_{l-1} \times\{e, \alpha\} \times \mathcal{T} \mathcal{B}_{(k+1, n)} .
$$

Consequently, $\left|C_{n}(\alpha)\right|=\left|\mathcal{T B}_{l-1}\right| \times|\{e, \alpha\}| \times\left|\mathcal{T B}_{(k+1, n)}\right|=2 F_{2 l-3} F_{2(n-k)-3}$.

Let $I_{n}=\left\{\alpha \in \mathcal{T} \mathcal{B}_{n}: C_{n}(\alpha)=\{e, \alpha\}\right\}$. Clearly, all non-identity elements in the set $\mathcal{T B}_{2}=\left\{e, \sigma_{1}\right\}$ belong to $I_{2}$, and those in the set $\mathcal{T} \mathcal{B}_{3}=\left\{e, \sigma_{1}, \sigma_{2}, \sigma_{1} \sigma_{2}, \sigma_{2} \sigma_{1}\right\}$ belong to $I_{3}$. In the following result, we completely characterize the set $I_{n}$ for $n \geq 4$.

Theorem 2.6. For $n \geq 4$, we have

$$
I_{n}=\left[\sigma_{1}, \sigma_{n-1}\right] \sqcup\left[\sigma_{1}, \sigma_{n-2}\right] \sqcup\left[\sigma_{2}, \sigma_{n-1}\right] \sqcup\left[\sigma_{2}, \sigma_{n-2}\right] .
$$

Proof. Theorem 1.1 states that an element $\alpha \in \mathcal{T} \mathcal{B}_{n} \backslash\{e\}$ with $\sigma_{i} \mid \alpha$ for all $1<i<n-1$ has a tiny centralizer $C_{n}(\alpha)=\{e, \alpha\}$. Therefore, $\alpha \in I_{n}$. If $\sigma_{i} \nmid \alpha$ for some $1<i<n-1$, then we can write $\alpha=\alpha_{1} \alpha_{2}$ such that $\alpha_{1} \in \mathcal{T} \mathcal{B}_{i}$ and $\alpha_{2} \in \mathcal{T} \mathcal{B}_{(i, n)}$. Without loss of generality, if $\alpha_{1}=e$, then $\alpha=\alpha_{2}$, which implies that $\sigma_{1} \alpha=\alpha \sigma_{1}$. Consequently, if neither $\alpha_{1}$ nor $\alpha_{2}$ is an identity, then $\alpha_{1} \alpha=\alpha \alpha_{1}$ and $\alpha_{2} \alpha=\alpha \alpha_{2}$. Hence, $\alpha \notin I_{n}$. Lemma 1.2 gives the following:

$$
\begin{aligned}
I_{n} & =\left\{\alpha \in \mathcal{T B}_{n}: \sigma_{i} \mid \alpha \text { for all } 1<i<n-1\right\} \\
& =\left\{\alpha \in \mathcal{T B}_{n}: \beta \mid \alpha \text { for any one } \beta \in\left[\sigma_{2}, \sigma_{n-2}\right]\right\} \\
& =\left[\sigma_{2}, \sigma_{n-2}\right] \sqcup\left[\sigma_{1}, \sigma_{n-2}\right] \sqcup\left[\sigma_{2}, \sigma_{n-1}\right] \sqcup\left[\sigma_{1}, \sigma_{n-1}\right] .
\end{aligned}
$$


Lemma 2.7. For $n \geq 2$, in the set of tiny braids $\mathcal{T} \mathcal{B}_{n}$, we have $\left|\left[\sigma_{1}, \sigma_{1+j}\right]\right|=$ $\left|\left[\sigma_{i}, \sigma_{i+j}\right]\right|=2^{j}$. Moreover, $\left|I_{n}\right|=9 \times 2^{n-4}$ for any $n \geq 4$.

Proof. Clearly, $\left|\left[\sigma_{1}, \sigma_{1+j}\right]\right|=\left|\left[\sigma_{i}, \sigma_{i+j}\right]\right|$. Furthermore, we use induction on $j$ to show that $\left|\left[\sigma_{1}, \sigma_{1+j}\right]\right|=2^{j}$.

For $j=1,\left[\sigma_{1}, \sigma_{2}\right]=\left\{\sigma_{1} \sigma_{2}, \sigma_{2} \sigma_{1}\right\}$; hence, $\left|\left[\sigma_{1}, \sigma_{2}\right]\right|=2$. Let $\left|\left[\sigma_{1}, \sigma_{j}\right]\right|=$ $2^{j-1}$. By Lemma 1.2, $\left[\sigma_{1}, \sigma_{1+j}\right]=\sigma_{1+j}\left[\sigma_{1}, \sigma_{j}\right] \sqcup\left[\sigma_{1}, \sigma_{j}\right] \sigma_{1+j}$. Therefore,

$\left|\left[\sigma_{1}, \sigma_{1+j}\right]\right|=\left|\sigma_{1+j}\left[\sigma_{1}, \sigma_{j}\right]\right|+\left|\left[\sigma_{1}, \sigma_{j}\right] \sigma_{1+j}\right|=\left|\left[\sigma_{1}, \sigma_{j}\right]\right|+\left|\left[\sigma_{1}, \sigma_{j}\right]\right|=2 \times 2^{j-1}=2^{j}$.

By Equation (2), we obtain

$$
\begin{aligned}
\left|I_{n}\right| & =\left|\left[\sigma_{1}, \sigma_{n-1}\right] \sqcup\left[\sigma_{1}, \sigma_{n-2}\right] \sqcup\left[\sigma_{2}, \sigma_{n-1}\right] \sqcup\left[\sigma_{2}, \sigma_{n-2}\right]\right| \\
& =\left|\left[\sigma_{1}, \sigma_{n-1}\right]\right|+\left|\left[\sigma_{1}, \sigma_{n-2}\right]\right|+\left|\left[\sigma_{2}, \sigma_{n-1}\right]\right|+\left|\left[\sigma_{2}, \sigma_{n-2}\right]\right| \\
& =2^{n-2}+2^{n-3}+2^{n-3}+2^{n-4} \\
& =4 \times 2^{n-4}+2 \times 2^{n-4}+2 \times 2^{n-4}+2^{n-4}=9 \times 2^{n-4} .
\end{aligned}
$$

For any $\alpha \in I_{n} \subset \mathcal{T} \mathcal{B}_{n},\left|C_{n}(\alpha)\right|=2$, and for every $\alpha \notin I_{n}$, we have the following result.

Theorem 2.8. For $n \geq 4, \min _{\alpha \notin I_{n}}\left|C_{n}(\alpha)\right|=4$ in $\mathcal{T} \mathcal{B}_{n}$.

Proof. Clearly, $\{e, \alpha\} \subseteq C_{n}(\alpha)$ for every $\alpha \in \mathcal{T} \mathcal{B}_{n}$. If $\alpha \notin I_{n}$, then by Lemma 2.6, $\sigma_{i} \nmid \alpha$ for some $1<i<n-1$; we can write $\alpha=\alpha_{1} \alpha_{2}$ such that $\alpha_{1} \in \mathcal{T H}_{i}$ and $\alpha_{2} \in \mathcal{T} \mathcal{B}_{(i, n)}$. Following the proof of Lemma 2.6, we can see that either $\left\{e, \alpha, \sigma_{1}, \sigma_{1} \alpha\right\} \subseteq C_{n}(\alpha),\left\{e, \alpha, \sigma_{n-1}, \sigma_{n-1} \alpha\right\} \subseteq C_{n}(\alpha)$ or $\left\{e, \alpha, \alpha_{1}, \alpha_{2}\right\} \subseteq C_{n}(\alpha)$. Therefore, $\left|C_{n}(\alpha)\right| \geq 4$ for all $\alpha \in \mathcal{T} \mathcal{B}_{n} \backslash I_{n}$. To obtain the required result, we need to prove the existence of an element $\beta \in$ $\mathcal{T B}_{n} \backslash I_{n}$ with $\left|C_{n}(\beta)\right|=4$. As $n \geq 4$, we can consider $\beta=\sigma_{1} \sigma_{2} \ldots \sigma_{n-3} \in$ $\mathcal{T} \mathcal{B}_{n}$, where $\beta \in\left[\sigma_{1}, \sigma_{n-3}\right]$. Hence, by Theorem 2.5,

$$
C_{n}(\beta)=\mathcal{T B}_{0} \times\{e, \beta\} \times \mathcal{T B}_{(n-2, n)}=\left\{e, \beta, \sigma_{n-1}, \beta \sigma_{n-1}\right\}
$$

Therefore, $\left|C_{n}(\beta)\right|=\left|\left\{e, \sigma_{n-1}, \beta, \beta \sigma_{n-1}\right\}\right|=4$.

The shifting property of Fibonacci numbers, i.e., $F_{m+n-1}=F_{m} F_{n}+$ $F_{m-1} F_{n-1}$, yields

$$
F_{m} F_{n} \leq F_{m+n-1} .
$$

This inequality enables us to find the maximum cardinality of $C_{n}(\alpha)$ for $\alpha \in\left[\sigma_{i}, \sigma_{i+j}\right]$.

Proposition 2.9. For all $n>3,\left|C_{n}\left(\sigma_{1}\right)\right|=\left|C_{n}\left(\sigma_{n-1}\right)\right|=2 F_{2 n-5}$, and for any other $\alpha \in\left[\sigma_{l}, \sigma_{k}\right] \subset \mathcal{T} \mathcal{B}_{n}, l \leq k<n, \max _{\alpha \in \mathcal{T} \mathcal{B}_{n}}\left|C_{n}(\alpha)\right|=2 F_{2 n-7}$. 
Proof. For $\alpha=\sigma_{1}$ or $\alpha=\sigma_{n-1}$, Theorem 2.5 yields $\left|C_{n}(\alpha)\right|=2 F_{2 n-5}$. For any other $\alpha \in\left[\sigma_{l}, \sigma_{k}\right] \subset \mathcal{T B}_{n}, l \leq k<n$, Theorem 2.5 implies $\left|C_{n}(\alpha)\right|=2 F_{2 l-3} F_{2(n-k)-3}$. By the Fibonacci number shifting property given in (3) and because $k-l \geq 0$, we obtain

$$
\left|C_{n}(\alpha)\right| \leq 2 F_{2 l-3+2(n-k)-3-1}=2 F_{2(n-(k-l))-7} \leq 2 F_{2 n-7} .
$$

\section{Properties of the Commuting Graph $\Gamma\left(\mathcal{T} \mathcal{B}_{n}\right)$}

The following characterization of the vertex set $V\left(\Gamma\left(\mathcal{T} \mathcal{B}_{n}\right)\right)$ follows from the definition of $\Gamma\left(\mathcal{T} \mathcal{B}_{n}\right)$ still be deleted to avoid being too repetitive or "wordy". and the proof of Lemma 2.6.

Lemma 3.1. A vertex $\alpha \in V\left(\Gamma\left(\mathcal{T} \mathcal{B}_{n}\right)\right)$ if and only if $\sigma_{i} \nmid \alpha$ for some $1<i<n-1$ and $\alpha \neq e$.

Lemma 2.7 enables us to find the number of vertices (order) of $\Gamma\left(\mathcal{T} \mathcal{B}_{n}\right)$.

Theorem 3.2. The order of $\Gamma\left(\mathcal{T} \mathcal{B}_{n}\right)$ is $F_{2 n-1}-9 \times 2^{n-4}-1$.

Proof. By definition, $V\left(\Gamma\left(\mathcal{T} \mathcal{B}_{n}\right)\right)=\mathcal{T H}_{n} \backslash\left(I_{n} \cup\{e\}\right)$ implies that

$$
\left|V\left(\Gamma\left(\mathcal{T} \mathcal{B}_{n}\right)\right)\right|=\left|\mathcal{T B}_{n}\right|-\left|I_{n}\right|-1=F_{2 n-1}-\left|I_{n}\right|-1 .
$$

By Lemma 2.7, $\left|V\left(\Gamma\left(\mathcal{T} \mathcal{B}_{n}\right)\right)\right|=F_{2 n-1}-9 \times 2^{n-4}-1$.

Proposition 3.3. For $n \geq 4, \Gamma\left(\mathcal{T} \mathcal{B}_{n}\right)$ is connected.

Proof. Consider two vertices $\alpha, \beta \in V\left(\Gamma\left(\mathcal{T} \mathcal{B}_{n}\right)\right)$. By Lemma 3.1, $\sigma_{i} \nmid \alpha$ for some $1<i<n-1$. Hence, we can write $\alpha=\alpha_{1} \alpha_{2}$ such that $\alpha_{1} \in \mathcal{T} \mathcal{B}_{i}$ and $\alpha_{2} \in \mathcal{T B}_{(i, n)}$. Similarly, $\sigma_{j} \nmid \beta$ for some $1<j<n-1$. Hence, $\beta=\beta_{1} \beta_{2}$ such that $\beta_{1} \in \mathcal{T} \mathcal{B}_{j}$ and $\beta_{2} \in \mathcal{T} \mathcal{B}_{(j, n)}$. We must consider two cases.

Case 1: If none of $\alpha_{1}, \alpha_{2}, \beta_{1} \beta_{2}$ is the identity, then for $i \leq j$, the path $\left(\left(\alpha, \alpha_{1}\right),\left(\alpha_{1}, \beta_{2}\right),\left(\beta_{2}, \beta\right)\right)$ exists, while for $i>j$, the path $\left(\left(\alpha, \alpha_{2}\right),\left(\alpha_{2}, \beta_{1}\right),\left(\beta_{1}, \beta\right)\right)$ exists.

Case 2: Without loss of generality, we suppose that $\alpha_{1}=e$, which implies that $\alpha=\alpha_{2}$. Consider $\beta_{2} \neq e$; then, we have the path $\left(\left(\alpha, \sigma_{1}\right),\left(\sigma_{1}, \beta_{2}\right),\left(\beta_{2}, \beta\right)\right)$. If $\beta_{2}=e$, then $\beta=\beta_{1}$, and we have the path $\left(\left(\alpha, \sigma_{1}\right),\left(\sigma_{1}, \sigma_{n-1}\right),\left(\sigma_{n-1}, \beta\right)\right)$. Consequently, $\Gamma\left(\mathcal{T} \mathcal{B}_{n}\right)$ is connected.

Since $\Gamma\left(\mathcal{T B}_{4}\right)=K_{3}$, the diameter of $\Gamma\left(\mathcal{T} \mathcal{B}_{4}\right)$ is 1 . For $n \geq 5$, we have the following result.

Theorem 3.4. For any $n \geq 5$, the diameter of $\Gamma\left(\mathcal{T B}_{n}\right)$ is 3 . 
Proof. The proof of Proposition 3.3 shows that $\Gamma\left(\mathcal{T} \mathcal{B}_{n}\right)$ has a diameter $\leq 3$. To show that it is exactly 3 , we show the existence of a path of length 3 between two vertices of $\Gamma\left(\mathcal{T} \mathcal{B}_{n}\right)$. Since $n \geq 5$, we consider $\alpha=\sigma_{2} \sigma_{3} \ldots \sigma_{n-3}, \beta=\sigma_{3} \sigma_{4} \ldots \sigma_{n-2} \in V\left(\Gamma\left(\mathcal{T} \mathcal{B}_{n}\right)\right)$, where, in fact, $\alpha \in$ $\left[\sigma_{2}, \sigma_{n-3}\right]$ and $\beta \in\left[\sigma_{3}, \sigma_{n-2}\right]$. Therefore, by Theorem 2.5,

$$
\begin{aligned}
& C_{n}(\alpha)=\mathcal{T B}_{1} \times\{e, \alpha\} \times \mathcal{T} \mathcal{B}_{(n-2, n)}=\left\{e, \alpha, \sigma_{n-1}, \alpha \sigma_{n-1}\right\} \\
& \text { and } C_{n}(\beta)=\mathcal{T} \mathcal{B}_{2} \times\{e, \beta\} \times \mathcal{T} \mathcal{B}_{(n-1, n)}=\left\{e, \beta, \sigma_{1}, \sigma_{1} \beta\right\}
\end{aligned}
$$

Clearly, the most likely shortest path between $\alpha$ and $\beta$ is $\left(\left(\alpha, \sigma_{n-1}\right),\left(\sigma_{n-1}, \sigma_{1}\right),\left(\sigma_{1}, \gamma\right)\right)$.

Theorem 3.5. For $\alpha \in V\left(\Gamma\left(\mathcal{T} \mathcal{B}_{n}\right)\right), n \geq 4, K_{3}$ is a subgraph of $\Gamma\left(\mathcal{T} \mathcal{B}_{n}\right)$ such that $\alpha \in V\left(K_{3}\right)$. Moreover, $\operatorname{girth}\left(\Gamma\left(\mathcal{T B}_{n}\right)\right)=3$.

Proof. By Lemma 3.1, $\sigma_{i} \nmid \alpha$ for some $1<i<n-1$. We can write $\alpha=\alpha_{1} \alpha_{2}$ such that $\alpha_{1} \in \mathcal{T} \mathcal{B}_{i}$ and $\alpha_{2} \in \mathcal{T} \mathcal{B}_{(i, n)}$. If $\alpha_{1}=e$, then $\alpha=$ $\alpha_{2} \in V\left(K_{3}\right)=\left\{\alpha, \sigma_{1}, \sigma_{1} \alpha\right\}$; a similar existence can be shown for $\alpha_{2}=e$. If neither $\alpha_{1}$ nor $\alpha_{2}$ is an identity, then $\alpha \in V\left(K_{3}\right)=\left\{\alpha, \alpha_{1}, \alpha_{2}\right\}$. Clearly, $\operatorname{girth}\left(\Gamma\left(\mathcal{T} \mathcal{B}_{n}\right)\right)=3$.

Based on the definition of the vertices of $\Gamma\left(\mathcal{T} \mathcal{B}_{n}\right)$, the identity element $\{e\}$ and element $\alpha \in V\left(\Gamma\left(\mathcal{T B}_{n}\right)\right)$ themselves do not contribute to the degree of $\alpha$, which gives $\operatorname{deg}(\alpha)=\left|C_{n}(\alpha)\right|-2$. This motivates us to discuss the degree-related properties of the commuting graph as a consequence of the results obtained in the previous section.

Proposition 3.6. For $\alpha \in\left[\sigma_{l}, \sigma_{k}\right] \subset \mathcal{T B}_{n}, l \leq k<n, n \geq 4$, we have

$$
\operatorname{deg}(\alpha)=2\left(F_{2 l-3} F_{2(n-k)-3}\right)-2 .
$$

Proof. It follows from Theorem 2.5.

Recall that $\left(F_{k}\right)$ is the Fibonacci sequence and that $F_{-1}=1$. Moreover, if $\operatorname{deg}(\alpha)=0$, then $\alpha \notin V\left(\Gamma\left(\mathcal{T} \mathcal{B}_{n}\right)\right)$.

By setting $l=k$ in Proposition 3.6, we obtain the following result.

Corollary 3.7. For $\sigma_{k} \in \mathcal{T} \mathcal{B}_{n}, n \geq 4$, we have

$$
\operatorname{deg}\left(\sigma_{k}\right)=2\left(F_{2 k-3} F_{2(n-k)-3}\right)-2 .
$$

The following Corollaries are obtained from Theorem 2.8 and Proposition 2.9 , respectively.

Corollary 3.8. For $n \geq 4$, the minimum degree $\delta\left(\Gamma\left(\mathcal{T} \mathcal{B}_{n}\right)\right)=2$.

Corollary 3.9. For any $n \geq 4, \operatorname{deg}\left(\sigma_{1}\right)=\operatorname{deg}\left(\sigma_{n-1}\right)=2 F_{2 n-5}-2$, and for any other $\alpha \in\left[\sigma_{l}, \sigma_{k}\right] \subset \mathcal{T} \mathcal{B}_{n}, l \leq k<n, \max (\operatorname{deg}(\alpha))=2 F_{2 n-7}-2$. 


\section{Open Problems}

The following questions related to $\Gamma\left(\mathcal{T} \mathcal{B}_{n}\right)$ remain to be explored.

Question 4.1. What is the number of edges (size) of $\Gamma\left(\mathcal{T} \mathcal{B}_{n}\right)$ ?

The degree of a certain class of vertices is discussed in Proposition 3.6.

Question 4.2. What is the degree of a general vertex in $\Gamma\left(\mathcal{T B}_{n}\right)$ ?

Proposition 3.6 shows that the degree of each $\alpha \in\left[\sigma_{i}, \sigma_{j}\right] \subset \mathcal{T} \mathcal{B}_{n}$ is even in $\Gamma\left(\mathcal{T B}_{n}\right)$.

Question 4.3. Is the degree of each vertex even in $\Gamma\left(\mathcal{T} \mathcal{B}_{n}\right)$ ?

\section{References}

[1] U. Ali, F. Azam, I. Javaid and A. Haider, Braids with trivial simple centralizer, Algebra Colloq., 22(4), 561-566 (2015)

[2] U. Ali and A. Haider, Centralizer of braids and Fibonacci numbers, Util. Math., 89, 289-296 (2012)

[3] E. Artin, Theory of braids. Ann. Math., 48, 101-126 (1947)

[4] R. Ashraf, B. Berceanu, Simple braids, arXiv:1003.6014v1 [math.GT] (2010)

[5] R. Ashraf, B. Berceanu, A. Riasat, Fibonacci numbers and positive braids, Ars Comb., 122, 299-306 (2015)

[6] C. Bates, D. Bondy, S. Perkins, P. Rowley, Commuting involution graphs for symmetric groups, J. Algebra, 266(1), 133-153 (2003)

[7] D. Bondy, The connectivity of commuting graphs, J. Combin. Theory (Ser. A), 113 995-1007 (2006)

[8] F. A. Garside, The braid groups and other groups, Quat. J. Math. Oxford, ser., 20, 235-254 (1969)

[9] A. Iranmanesh, A. Jafarzadeh, On the commuting graph associated with the sym- metric and alternating groups, J. Algebra Appl., 7 (1) 129-146 (2008)

[10] C. Kassel and V. Turaev, Braid Groups. Grad. Texts in Math., Springer, New York, (2008)

[11] D. B. West, Introduction to Graph Theory. Prentice Hall, (1996) 